\title{
CISTITIS AGUDA, PROSTATITIS Y URETRITIS
}

\section{ACUTE CYSTITIS, PROSTATITIS AND URETHRITIS}

\author{
Guillermo De'Marziani ${ }^{1}$
}

\section{RESUMEN}

La cistitis bacteriana aguda es un proceso infeccioso de la vejiga. La prescencia de disuria, polaquiuria, tenesmo, dolor hipogástrico y/o hematuria, aumenta la probabilidad diagnóstica. Ante la sospecha clínica debe solicitarse sedimento urinario y evaluar presencia de leucocituria, piuria o microhematuria, y requerir urocultivo previo al inicio del tratamiento. La prostatitis bacteriana aguda es una entidad poco frecuente pero que requiere diagnóstico y tratamiento precoz, con aislamiento del gérmen. La uretritis puede ser de etiología infecciosa o no. En estas patologías, el tratamiento no difiere de los pacientes sin diabetes, pero deben tenerse presente factores como la neuropatía autonómica que puede encubrir la sintomatología y aumentar el riesgo de descompensación metabólica y cetoacidosis.

Palabras clave: cistitis; prostatitis; uretritis; aislamiento bacteriano; neuropatía autonómica.

Revista de la Sociedad Argentina de Diabetes 2019; Vol. 53 (28-50)

\section{ABSTRACT}

Acute bacterial cystitis is an infectious process of the bladder. The presence of dysuria, pollakiuria, tenesmus, hypogastric pain and/or hematuria increases the diagnostic probability. In view of clinical suspicion, urinary sediment should be requested to evaluate the presence of leukocyturia, pyuria or microhematuria, and urine culture requested before starting treatment. Acute bacterial prostatitis is a less frequent entity but requires early diagnosis and treatment, with germ isolation. Urethritis can be of infectious etiology or not. In these pathologies, treatment does not differ from patients without diabetes, but factors such as autonomic neuropathy that can conceal the symptoms and increase the risk of metabolic decompensation and ketoacidosis should be considered.

Key words: cystitis; prostatitis; urethritis; bacterial isolation; autonomic neuropathy.

Revista de la Sociedad Argentina de Diabetes 2019; Vol. 53 (28-50)
Médico especialista en Medicina Interna, especialista en Nefrología y Magister en Diabetes en el Centro de Enfermedades Renales e Hipertensión Arterial (CEREHA S.A.), Sarandí, Provincia de Buenos Aires, Argentina

\author{
Contacto del autor: Guillermo De'Marziani \\ E-mail: guille_dm@hotmail.com \\ Correspondencia: Malabia $24703^{\circ}$ A, (C1425EZJ), Ciudad \\ Autónoma de Buenos Aires, Argentina \\ Fecha de trabajo recibido: 25/03/19 \\ Fecha de trabajo aceptado: 03/04/19
}

Conflictos de interés: el autor declara que no existe conflicto de interés.

\section{Cistitis}

La cisititis bacteriana aguda es una entidad que se presenta como consecuencia de un proceso infeccioso en la vejiga. Es más frecuente en el género femenino que masculino y más aún en mujeres sexualmente activas, y se estima que una de cada tres mujeres presentarán al menos una infección del tracto urinario (ITU) diagnosticada con requerimiento de tratamiento antibiótico, mientras que de un 40 a un $50 \%$ padecerán una ITU en algún momento de su vida'. En un estudio en mujeres jóvenes sexualmente activas que iniciaron un nuevo método anticonceptivo se evidenció una incidencia de 0,7 episodios/persona/año contrastando en lo observado en la población de mujeres postmenopáusicas con una incidencia de 0,07 episodios/persona/año². En mujeres jóvenes, la recurrencia puede alcanzar hasta el $25 \%$ dentro de los seis meses luego del primer episodio y la tasa de recurrencia podrá ser de 0,73 episodios/ paciente/año luego del primer episodio ${ }^{3}$.

En un estudio de 1.350 .000 pacientes, en 2012, se detectó un incremento del riesgo del $60 \%$ para desarrollo de ITU en la población diabética vs no diabética. La tasa de incidencia estimada fue de 46.9/1.000 pacientes/año (PA) (95\% Cl 45,8-48,1/1.000 PA) en personas con diabetes y $29,9 / 1.000 \mathrm{PA}(95 \% \mathrm{Cl}$ 28,9-30,8/1.000 PA) sin diabetes ${ }^{4}$.

La glucosuria como factor de riesgo de ITU continua aún es controversial; en pacientes con glucosuria renal familiar no se encuentra incremento del riesgo de infecciones urogenitales, por lo cual la glucosuria per se no incrementaría el riesgo ${ }^{5}$. La modificación de la función del sistema inmune, entre las que destacan la alteración de la migración, la muerte intracelular, fagocitosis o quimiotaxis de polimorfonucleares, puede debilitar la respuesta del huésped contra las noxas infeccio- 
sas en presencia de diabetes (DM). La neuropatía autonómica genitourinaria, que altera el adecuado vaciado vesical y genera retención de orina disminuyendo el barrido físico bacteriano durante la micción, favorece la proliferación bacteriana. La disfunción vesical se encuentra presente en un 26-85\% de las mujeres con DM, dependiendo del tiempo de evolución de la neuropatía, así como de la duración de la enfermedad metabólica ${ }^{6}$.

Los síntomas y signos son disuria, polaquiuria, tenesmo, dolor hipogástrico y/o hematuria. La probabilidad de cistitis es mayor del 50\% cuando se encuentra presente alguno de los síntomas mencionados y mayor del $90 \%$ con disuria y polaquiuria en ausencia de flujo o irritación vaginal?. Los pacientes con DM pueden tener presentaciones más sintomáticas (fiebre, síntomas sistémicos) o muy leves inicialmente como en caso de neuropatía autonómica. En presencia de hiper/hipoglucemias o estado hiperosmolar o cetoacidosis es necesario realizar diagnóstico temprano de procesos infecciosos, entre ellos la ITU.

Ante la sospecha clínica de cistitis debe solicitarse muestra de orina de chorro medio para sedimento urinario y evaluar presencia de leucocituria, piuria o microhematuria; es importante solicitar urocultivo previo al inicio del tratamiento dado que pueden presentarse patógenos resistentes como enterobacterias $B L E E+$, uropatógenos resistentes a quinolonas, enterobacterias productoras de carbapenemasas o enterococos vancomicina resistentes ${ }^{8}$. Esta condición es frecuente por el uso de múltiples esquemas de antibióticos en situaciones como bacteriuria asintomática (BA) o síntomas irritativos de vía urogenital. Podría realizarse tratamiento antibiótico empírico, sin urocultivo inicial si existen síntomas sugerentes de cistitis aguda en mujeres con DM que no presentan complicaciones crónicas u otra complicación estructural urológica.

El tratamiento inicial no difiere entre pacientes con o sin DM y debe ser adecuado según la sensibilidad obtenida en la identificación del germen y antibiograma?.

\section{Prostatitis}

Refiere a un conjunto de síndromes clínicos, entre los que se incluyen infecciones bacterianas agudas y crónicas, así como el pobremente definido síndrome de dolor pelviano crónico y la inflamación asintomática de la glándula prostática que se encuentra en piezas de anatomía patológica. A diferencia de la hipertrofia prostática benigna y el cáncer de próstata que se manifiestan predominantemente en adultos mayores, la prostatitis puede presentarse en hombres de todas las edades. Se la considera una afección del tracto genitourinario en su conjunto y se estima una prevalencia de hasta un $16 \%^{10}$. Si bien la prostatitis bacteriana aguda primaria es una entidad poco común (sólo 4\% de casos de prostatitis), los procesos infecciosos agudos del tracto urinario en hombres pueden comprometer en hasta un $90 \%$ de los casos el tejido prostático y si se considera que el incremento del riesgo de ITU en hombres con DM es casi un $50 \%$ con respecto a sin diabetes, es frecuentemente subdiagnosticada y no considerada en los probables diagnósticos diferenciales ${ }^{11}$. Además se afecta significativamente la calidad de vida debido a las complicaciones asociadas como disfunción eréctil, eyaculación precoz y alteraciones en el deseo sexual.

El síndrome de inflamación prostática se divide en cuatro categorías como lo propone la clasificación del National Institutes of Health $(\mathrm{NIH})^{12}$ (Tabla 1). La prostatitis aguda primaria afecta a hombres a partir de los 20 años, con un pico de incidencia en los mayores de 60 años. Los factores de riesgo, debido a que se favorece la vía ascendente de diseminación, incluyen trastornos estructurales de la vía urinaria, uso de catéteres uretrales (cateterismos intermitentes están relacionados con hasta un 33\% de los episodios), instrumentación de la vía urinaria (cistouretroscopía, biopsia prostática transrectal), actividad sexual no protegida e hipertrofia prostática benigna. La infección vía linfática desde el recto o hematógena es muy rara.

Los signos y síntomas pueden ser: dolor hipogástrico y en región perineal, síntomas infecciosos (fiebre, escalofríos, vómitos, astenia) y síntomas relacionados con la vía urinaria (disuria, tenesmo, polaquiuria $)^{13}$. En casos severos pueden presentarse síntomas y signos de sepsis. El examen físico refleja dolor hipogástrico intenso, con signos relacionados con la presencia de orquiepididimitis. Debe obtenerse, sin excepción, una muestra de orina para sedimento urinario y urocultivo. El masaje prostático se encuentra contraindicado por el riesgo de diseminación bacteriana hematógena; el tratamiento antibiótico inicialmente es empírico ${ }^{14}$.

\section{Uretritis}

Proceso inflamatorio de la uretra debido a agentes infecciosos y no infecciosos. Desde el punto de vista clínico y terapéutico, a las causas infecciosas 
se las clasifica de acuerdo a los agentes causales como uretritis no gonocócicas (UNG) y uretritis gonocócicas (UG). Si bien en Europa Central y EE.UU. la etiología más común es la UNG, hasta en un $50 \%$ de los casos no se detecta el agente involucrado ${ }^{15}$. Existe una correlación entre la actividad sexual, el nivel socioeconómico bajo y la frecuencia de infecciones debidas a Neisseria gonorrhoeae (NG) y Chlamydia trachomatis (CT).

Se considera a CT como el agente más comúnmente identificado en UNG, seguida por Mycoplasma genitalium (MG). En aproximadamente la mitad de los casos no puede determinarse la etiología, no obstante algunos estudios sugieren que Leptotrichia/Sneathia deberían considerarse en caso de antecedentes de prácticas homosexuales en hombres ${ }^{16}$.

Los síntomas se hacen presentes luego de un período de incubación de 4-7 días para UG y 5-8 días para UNG. La disuria es el signo más representativo en la mayoría de los casos de UG y en la mitad de UNG, pudiendo acompañarse de prurito, sensación de quemazón y secreción uretral, ya sea mucosa, acuosa o francamente purulenta, en la primera orina de la mañana o a lo largo del día. Algunos procesos pueden cursar en forma asintomática.

El diagnóstico se debe confirmar en un paciente sintomático con alguno de los siguientes criterios ${ }^{17}$ :

- Secreción uretral mucopurulenta o purulenta.

- Presencia de $\geq 2$ leucocitos por campo de inmersión en aceite de hisopado uretral.

- Presencia de >10 leucocitos por campo (x1.000 aumentos) de primera porción de orina matinal, sin aseo previo.

Algunos laboratorios utilizan test de amplificación de ácidos nucleicos (NAAT) en la primera orina matinal para detectar CT y NG, los cuales presentan mayor sensibilidad, especificidad y facilidad para el transporte de la muestra. Las técnicas de GRAM pueden ser de alto valor si se detectan diplococos gram negativos intracelulares en las muestras, lo que confirma el diagnóstico de UG.

Las recomendaciones sugieren que debería confirmarse el agente etiológico con técnicas de NAAT en todos los pacientes que manifiesten síntomas con o sin signos de laboratorio de inflamación uretral.

El tratamiento inicial es empírico y debe indicarse en todo paciente con sospecha o confirmación de uretritis. El esquema dependerá de la evidencia clínica o de laboratorio de UG vs UNG (sospecha de contacto con individuo con gonorrea, gram con diplococos intracelulares). Cuando no se encuentra disponible la evaluación microscópica en personas sexualmente activas debería iniciarse esquema antibiótico para UG y UNG, siendo la cobertura inicial para CT por ser el agente etiológico más probable.

Para reducir el riesgo de transmisión y reinfección deberá indicarse abstinencia sexual hasta completar el tratamiento y realizar test para otras enfermedades de transmisión sexual $(\mathrm{VIH}$, sífilis) al paciente y la pareja sexual que haya tenido contacto hasta 60 días previos al diagnóstico. Para aquellos casos de uretritis por MG no existen recomendaciones formales en cuanto a los contactos, pero se considera razonable actuar de la misma manera que para los otros agentes.

\begin{tabular}{|l|l|}
\hline \multicolumn{2}{|c|}{ Clasificación de prostatitis NIH } \\
\hline Categoría & Diagnóstico \\
\hline I & Prostatitis bacteriana aguda \\
II & Prostatitis bacteriana crónica \\
& Prostatitis crónica/śndrome de \\
& dolor pelviano crónico (PC/SDPC) \\
& a) Inflamatorio \\
\hline IV & b) No inflamatorio \\
\hline
\end{tabular}

Adaptado de: Wagenlehner et al.

Tabla 1: Clasificación de prostatitis del National Institutes of Health.

\section{BIBLIOGRAFÍA}

1. Foxman B, Barlow R, D'Arcy $\mathrm{H}$, et al. Urinary tract infection: self-reported incidence and associated costs. Ann Epidemiol 2000; 10(8):509-15.

2. Hooton TM, Scholes D, Hughes JP, et al. A prospective study of risk factors for symptomatic urinary tract infection in young women. N Engl J Med 1996; 335(7):468-74.

3. Ikäheimo R, Siitonen A, Heiskanen, et al. Recurrence of urinary tract infection in a primary care setting: analysis of a 1-year follow-up of 179 women. Clin Infect Dis 1996; 22(1):91-99.

4. Hirji I, Guo Z, Andersson SW, et al. Incidence of urinary tract infection among patients with type 2 diabetes in the UK General Practice Research Database (GPRD). J Diabetes Complications 2012; 26(6):513-6.

5. Rizzi M, Trevisan R. Genitourinary infections in diabetic patients in the new era of diabetes therapy with sodium-glucose cotransporter-2 inhibitors. Nutr Metab Cardiovasc Dis 2016; 26(11):963-970.

6. Frimodt-Møller C. Diabetic cystopathy: epidemiology and related disorders. Ann Intern Med 1980; 92(2 Pt 2):318-21.

7. Bent $S$, Nallamothu BK, Simel D, et al. Does this woman have an acute uncomplicated urinary tract infection? JAMA 2002; 287(20):2701-10. 
8. Nitzan O, Elias M, Chazan B, Saliba W. Urinary tract infections in patients with type 2 diabetes mellitus: review of prevalence, diagnosis, and management. Diabetes Metab Syndr Obes 2015; 8:129-36

9. Gupta K, Hooton TM, Naber KG, et al. Infectious Diseases Society of America; European Society for Microbiology and Infectious Diseases. International clinical practice guidelines for the treatment of acute uncomplicated cystitis and pyelonephritis in women: a 2010 update by the Infectious Diseases Society of America and the European Society for Microbiology and Infectious Diseases. Clin Infect Dis 2011; 52(5):e103-20.

10. Collins MM, Meigs JB, Barry MJ, et al. Prevalence and correlates of prostatitis in the health professionals follow-up study cohort. J. Urol. 2002; 167(3): 1363-6.

11. Ulleryd P. Febrile urinary tract infection in men. Int $\mathrm{J}$ Antimicrob Agents 2003; Suppl 2:89-93.

12. Wagenlehner FM, Pilatz A, BschleipferT, et al. Bacterial prostatitis. World J Urol 2013; 31(4):711-6.
13. Etienne M, Chavanet $P$, Sibert $L$, et al. Acute bacterial prostatitis: heterogeneity in diagnostic criteria and management. Retrospective multicentric analysis of 371 patients diagnosed with acute prostatitis. BMC Infect Dis 2008; 8:12.

14. Grabe M, Bjerklund-JohansenTE, Botto H, Wullt B, et al. Guidelines on urological infections. European Association of Urology Guidelines, 2012 Edition. European Association of Urology, Arnhem, The Netherlands, 1-110.

15. Wetmore CM, Manhart LE, Lowens MS, et al. Demographic, behavioral, and clinical characteristics of men with nongonococcal urethritis differ by etiology: a case-comparison study. SexTransm Dis 2011; 383(3): 180-6.

16. Lyss SB, Kamb ML, Peterman TA; Project RESPECT Study Group, et al. Chlamydia trachomatis among patients infected with and treated for Neisseria gonorrhoeae in sexually transmitted disease clinics in the United States. Ann Intern Med 2003; 139(3):178-85.

17. Workowski KA, Bolan GA, et al. Sexually transmitted diseases treatment guidelines, 2015. Centers for Disease Control and Prevention, MMWR Recomm Rep 2015; 64(RR-03):1.

\section{PIELONEFRITIS Y NEFRITIS INTERSTICIAL}

\section{PYELONEPHRITIS AND INTERSTITIAL NEPHRITIS}

\author{
Gabriela Victoria Medek ${ }^{1}$
}

\section{RESUMEN}

La pielonefritis aguda es una infección frecuente que puede progresar a absceso o pielonefritis enfisematosa (PE); la mayoría de los casos se produce por Escherichia coli. La presentación clínica puede oscilar desde síntomas leves hasta septicemia. La posibilidad de bacteremia depende de factores del huésped: pacientes gravemente enfermos, inmunocomprometidos, con obstrucción del tracto urinario y mayores de 65 años. Más del $90 \%$ de PE se presenta en pacientes con diabetes. La nefritis intersticial afecta el intersticio renal, puede ser aguda o crónica, la mayoría de las veces debido a AINES o antibióticos. Ambas patologías son predominantes en mujeres.

Palabras clave: pielonefritis; pielonefritis enfisematosa; septicemia; nefritis intersticial.

Revista de la Sociedad Argentina de Diabetes 2019; Vol. 53 (28-50)

\section{ABSTRACT}

Acute pyelonephritis is a frequent infection that can progress to abscess or emphysematous pyelonephritis (EP); most cases are caused by Escherichia coli. The clinical onset can range from mild symptoms to septicemia. The possibility of bacteremia depends on host factors: patients who are seriously ill, immunocompromised, with urinary tract obstruction and $>65$ years of age. More than $90 \%$ of EP occurs in patients with diabetes. Interstitial nephritis affecting the renal interstitium, can be acute or chronic, in most cases due to NSAIDs or antibiotics. Both pathologies are predominant in women.

Key words: pyelonephritis; emphysematous pyelonephritis; septicemia; interstitial nephritis.

Revista de la Sociedad Argentina de Diabetes 2019; Vol. 53 (28-50)
Médica especialista en Medicina Interna especializada en Diabetes y Nutrición, Servicio de Diabetes y Nutrición, Policlínica Bancaria, La Plata, Provincia de Buenos Aires, Argentina
Contacto de la autora: Gabriela Victoria Medek

E-mail: gmedek@hotmail.com

Correspondencia: Acevedo 840, $5^{\circ}$ piso, Depto 6, (C1414DJR), Ciudad Autónoma de Buenos Aires, Argentina

Fecha de trabajo recibido: 25/03/19

Fecha de trabajo aceptado: 03/04/19

Conflictos de interés: la autora declara que no existe conflicto de interés. 